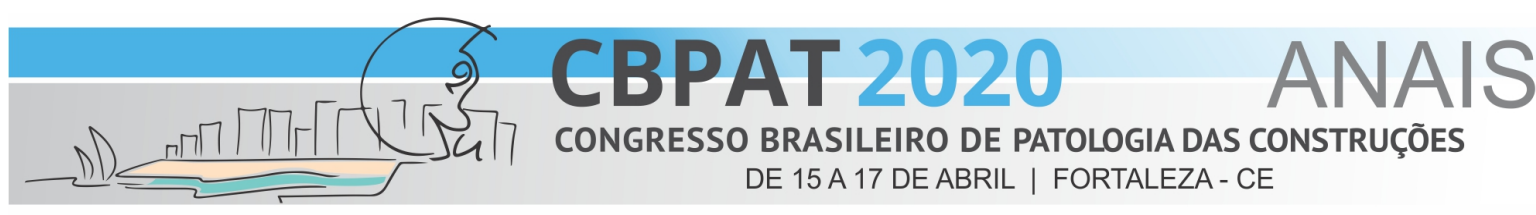

http://dx.doi.org/10.4322/CBPAT.2020.025

\title{
REFORÇO À FLEXÃO DE VIGAS BIAPOIADAS EM CONCRETO ARMADO COM BARRAS DE FIBRA DE VIDRO
}

\author{
VARES, RHAISSA \\ Engenheira Civil \\ Universidade Federal do Rio Grande do Sul \\ Rio Grande do Sul; Brasil \\ rhaisv@gmail.com
}

LAZZARI, PAULA

Professora Doutora

Universidade Federal do Rio Grande do Sul

Rio Grande do Sul; Brasil

p.manica.lazzari@gmail.com

MARTINI, ANDREW

Bolsista de Iniciação Científica

Universidade Federal do Rio Grande do Sul

Rio Grande do Sul; Brasil

andrewwmartini@gmail.com

\author{
HASTENPFLUG, DANIEL \\ Professor Doutor \\ Universidade Federal de Santa Catarina \\ Santa Catarina; Brasil \\ hasten@gmail.com \\ LAZZARI, BRUNA \\ Professora Mestre \\ Pontifícia Universidade Católica do RS \\ Rio Grande do Sul; Brasil \\ bruna.ml@gmail.com
}

\section{RESUMO}

Materiais compósitos vem sendo cada vez mais estudados pela comunidade acadêmica, em decorrência da otimização de suas propriedades mecânicas. As barras de fibra de vidro, formadas por GFRP (Glass Fiber Reinforced Polymer), possuem elevada resistência à corrosão, neutralidade eletromagnética e capacidade isolante térmica e elétrica, o que as tornam uma boa opção ao aço no uso em armaduras para o concreto armado. Desta maneira, estudou-se, de forma analítica e experimental, o comportamento de barras de GFRP em reforços estruturais de vigas biapoiadas de concreto armado submetidas à flexão simples, visando aumentar a capacidade de carga destes elementos estruturais. Para isto, moldaram-se oito vigas em concreto armado, sendo duas vigas referência e seis vigas reforçadas. Entre as vigas reforçadas, foram analisadas duas para cada diâmetro de barra de GFRP ( $8 \mathrm{~mm}, 10 \mathrm{~mm}$ e $12 \mathrm{~mm}$ ), utilizadas como reforço estrutural. A partir do estudo experimental, foi possível traçar as curvas carga x deslocamento e as curvas carga $\mathrm{x}$ deformação da armadura obtidas a partir dos strain gages. Os resultados encontrados mostram que os momentos últimos das vigas reforçadas com fibra de vidro observados de maneira experimental foram inferiores aos determinados de maneira teórica, o que reforça a necessidade de um estudo mais específico para materiais GFRP.

\section{Palavras-chave: GFRP, barras de fibra de vidro, reforço estrutural.}

\begin{abstract}
Composite materials have been increasingly studied by the academic community due to the optimization of their mechanical properties. Glass Fiber Reinforced Polymer (GFRP) bars have high corrosion resistance, electromagnetic neutrality and thermal and electrical insulating capabilities, making them a good choice for steel when using reinforced concrete reinforcement. Thus, an analytical and experimental study was done to know the behavior of GFRP bars in reinforced concrete beams submitted to simple bending, aiming to increase the load capacity of these structural elements. For this, eight reinforced concrete beams were molded, being two reference beams and six reinforced beams. Among the reinforced beams, two were analyzed for each GFRP bar diameter $(8 \mathrm{~mm}, 10 \mathrm{~mm}$ and $12 \mathrm{~mm})$, used as structural reinforcement. From the experimental study it was possible to trace the load $\mathrm{x}$ displacement curves and the load $\mathrm{x}$ deformation curves obtained from strain gages. The results show that the maximum moment of the experimental study in the glass fiber reinforced beams were lower than the theoretical ones, which reinforces the need for a more specific study for GFRP materials.
\end{abstract}

Keywords: GFRP, fiberglass bars, structural reinforcement. 


\section{INTRODUÇÃO}

A fim de restaurar a segurança e aumentar a durabilidade das construções, tornou-se comum a utilização de técnicas capazes de restabelecer condições estruturais e de uso adequadas das estruturas. Entretanto, a necessidade de se realizar intervenções pode estar atrelada a fatores que não são unicamente a qualidade e durabilidade da construção, mas sim à mudança de uso que essa possa sofrer. Dessa forma, técnicas de reforços estruturais surgem como alternativa para solucionar essas questões.

A utilização de barras feitas com fibras reforçadas com polímeros vem conquistando maior destaque na engenharia civil, em decorrência de seu baixo peso, sua capacidade isolante térmica, suas elevadas resistência mecânica e resistência à corrosão e, no caso das fibras de vidro, ainda é possível ressaltar que possuem neutralidade eletromagnética, o que as diferem dos polímeros com fibras de carbono (ACI 440.2R-02, 2002). Contudo, apesar dos pontos positivos que esses materiais oferecem, os profissionais da área de engenharia ainda contam com, basicamente, técnicas fundadas em experiências empíricas anteriores e com poucas normas específicas de projeto de reforço com materiais compósitos, o que dificulta a inserção na construção civil. Desta maneira, evidencia-se a importância de estudos experimentais e computacionais em relação ao tema.

O uso da fibra de vidro, de forma difusa em concretos e argamassas, é feito para se aumentar a capacidade de deformação desses materiais e auxiliar na capacidade de resistir maiores solicitações de tração, de solicitações compostas e impacto. Atualmente, também tem sido utilizada com o intuito de controlar a fissuração, decorrentes do processo de hidratação, do cimento em concretos e argamassas (PERUZZI, 2002).

O emprego das fibras em formas de barras, tecidos e telas surge como alternativa a armaduras de aço, por essas estarem sujeitas à corrosão (PERUZZI, 2007). Entretanto, para que se possa, de fato, serem feitas as substituições dos materiais metálicos, é necessário realizar uma investigação experimental aprofundada em relação ao seu comportamento frente às deformações estruturais, resistência à tração, ruptura, entre outras. Um dos aspectos que tem sido debatido na comunidade acadêmica é a susceptibilidade das fibras em meios alcalinos que, com o passar do tempo, provocam perdas nas propriedades físicas como a redução da resistência à tração (PERUZZI, 2007).

Entre as alternativas de uso das barras de GFRP (Glass Fiber Reinforced Polymer) está o reforço de estruturas submetidas à flexão. De acordo com Couto (2007) é interessante ressaltar que as propriedades físicas e mecânicas das barras influenciam na transferência de tensões ao material cimentício, sendo esse um dos pontos de estudo nesse trabalho.

\section{PROGRAMA EXPERIMENTAL}

O programa experimentalconstituiu-se em ensaiar vigas em concreto armado à flexão elaboradas e reforçadas em laboratório. O estudo analisou o comportamento físico de barras de fibra de vidro, de três diferentes diâmetros, quando utilizadas para o reforço à flexão de vigas biapoiadas. Para a realização desse trabalho foram ensaiadas experimentalmente oito vigas em concreto armado, sendo seis reforçadas com barras de fibra de vidro de $8 \mathrm{~mm}$ (VR1 e VRR1), $10 \mathrm{~mm}$ (VR2 e VRR2) e $12 \mathrm{~mm}$ (VR3 e VRR3) e duas vigas testemunho (VT e VT1). A adoção desses diâmetros para a execução do reforço fundou-se na disponibilidade do mercado. A Tabela 1 apresenta a descrição do programa experimental.

\begin{tabular}{cc} 
Tabela 1 - Descrição do programa experimental \\
\hline Vigas & $\begin{array}{c}\text { Ф (barra } \\
\text { GFRP) }\end{array}$ \\
\hline VT, VT1 & - \\
\hline VR1, VRR1 & $8(\mathrm{~mm})$ \\
\hline VR2, VRR2 & $10(\mathrm{~mm})$ \\
\hline VR3, VRR3 & $12(\mathrm{~mm})$ \\
\hline
\end{tabular}

\subsection{Característica das vigas}

Foram ensaiadas vigas de concreto armado com comprimento de $150 \mathrm{~cm}$, largura de $10 \mathrm{~cm}$ e altura de $15 \mathrm{~cm}$. Todas as vigas foram armadas com duas barras de aço CA-50 de $8 \mathrm{~mm}$ na zona tracionada e duas barras de aço CA-60 de 6,3 mm na zona comprimida. Todas as vigas receberam 16 estribos de aço CA-60 de $5 \mathrm{~mm}$ distribuídos igualmente nos dois terços externos das vigas com espaçamento de $7 \mathrm{~cm}$. A tensão de escoamento das barras de aço, obtida por ensaio de 


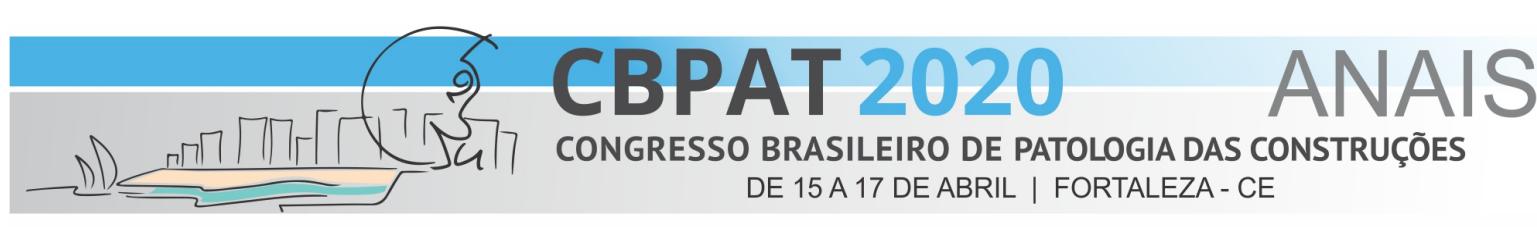
ISBN 978-65-86819-05-2 DE PATOLOGRIALIRA CONSTRUÇOES

tração, foi de $670 \mathrm{MPa}$. Para o concreto, realizou-se ensaio para a determinação da resistência à compressão e o módulo de elasticidade, aos 28 dias, obtendo-se os valores médio de 42,05 MPa e 39,44 GPa. A Figura 1 mostra o detalhe das armaduras das vigas.

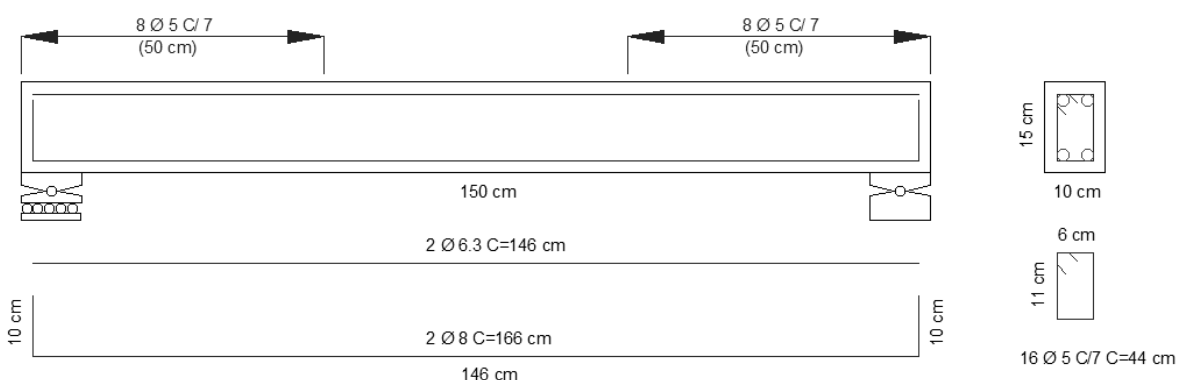

Figura 1 - Detalhe da geometria das vigas

\subsection{Característica do sistema de reforço}

A metodologia de verificação das vigas baseou-se nos princípios e hipóteses do estado limite último, considerando apenas a ruptura por flexão, por esmagamento do concreto na zona comprimida ou falha das barras de fibra de vidro à tração. Neste estudo não foram considerados os coeficientes de majoração de carga e de minoração da resistência dos materiais, assim como o efeito Rush de carregamentos de longa duração.

A camada de reforço foi executada em argamassa estrutural e possui dimensões $126 \mathrm{~cm}$ de largura e 2,5 cm de altura, com as armaduras de fibra de vidro aplicadas diretamente no fundo das vigas. Realizou-se teste de resistência à compressão, nas idades 14 e 15 dias, para a argamassa estrutural. Devido à trabalhabilidade do material ser baixa, executou-se uma betonada por viga, o que resultou em seis valores de resistência média à compressão, conforme se observa na Tabela 2.

Tabela 2 - Resistência média à compressão da argamassa estrutural das vigas reforçadas

\begin{tabular}{cccc}
\hline Vigas & Idade (em dias) & Resistência média à compressão (MPa) & Desvio-padrão (MPa) \\
\hline VR1 & 15 & 63,08 & 1,89 \\
\hline VR2 & 15 & 60,96 & 13,41 \\
\hline VR3 & 15 & 71,35 & 7,92 \\
\hline VRR1 & 15 & 60,38 & 16,64 \\
\hline VRR2 & 14 & 62,71 & 9,96 \\
\hline VRR3 & 14 & 53,75 & 14,63 \\
\hline
\end{tabular}

A tensão de ruptura das barras de vidro não foi testada laboratorialmente, sendo os valores utilizados na verificação, obtidos por interpolação linear, a partir dos dados fornecidos pelo fabricante. Encontrou-se para as barras de diâmetro 8 $\mathrm{mm}, 1054,67 \mathrm{MPa}$, para as barras de $10 \mathrm{~mm}, 1027,67 \mathrm{MPa}$ e para as barras de $12 \mathrm{~mm}, 989 \mathrm{MPa}$. O módulo de elasticidade adotado para as barras, igualmente, foi o valor fornecido pelo fabricante de 48,44 GPa.

Seriam utilizados estribos de fibra de vidro para realizar a ancoragem da camada de reforço, entretanto não foi possível executar essa técnica dentro do cronograma do estudo. Sendo assim, para evitar o escorregamento das barras de fibra de vidro e o descolamento do reforço, durante o ensaio de flexão, utilizou-se uma camada de manta de fibra de vidro de largura $35 \mathrm{~cm}$ e comprimento $55 \mathrm{~cm}$, aplicada nos dois terços médios das vigas, de modo que ficassem $12 \mathrm{~cm}$ livres para o posicionamento nos apoios do teste. A preparação da superfície foi realizada com resina primer e a fixação da manta, com resina laminação. A Figura 2 apresenta o detalhe das vigas reforçadas. 


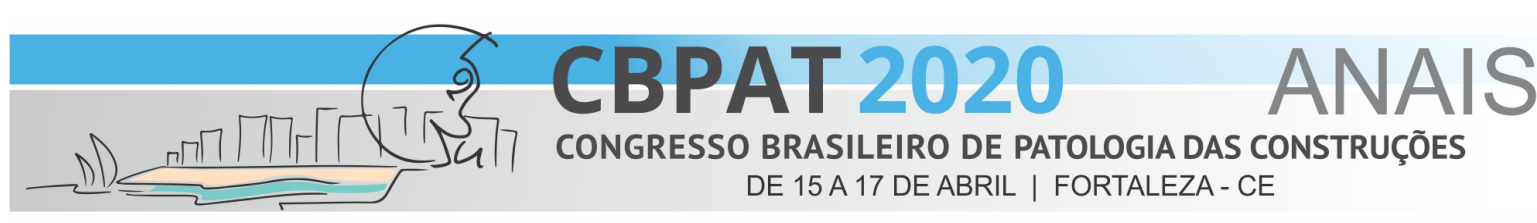

ISBN $978-65-86819-05-2$ ASSOCIACÃO BRASILEIRA DE PATOLOGIA DAS
CONSTRUÇOES

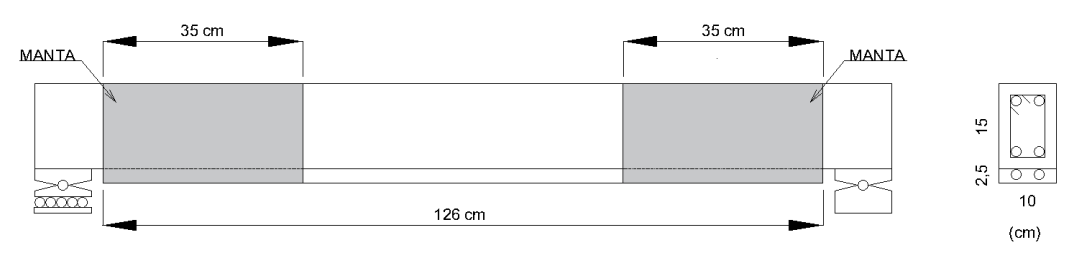

Figura 2 - Detalhe das vigas reforçadas

\subsection{Ensaio das vigas}

As vigas foram ensaiadas à flexão em quatro pontos gerando uma zona de momento fletor constante na região central dos elementos. Para a execução do ensaio, utilizou-se prensa de ensaios universal, com capacidade de $2000 \mathrm{kN}$ da marca Shimadzu. Como não existe uma Norma que apresente as diretrizes a se seguir nesse ensaio, utilizou-se os mesmos valores adotados por Nora (2018). Até os primeiros $15 \mathrm{~mm}$ de flecha, a taxa de carregamento foi de $1 \mathrm{~mm} / \mathrm{min}$. A partir de então, a taxa de carregamento foi aumentada para $5 \mathrm{~mm} / \mathrm{min}$. Essa metodologia foi adotada, em função da variação da flecha ser muito pequena nos instantes iniciais. A adoção inicial da taxa de $1 \mathrm{~mm} / \mathrm{min}$ foi na tentativa de se obter mais dados para análise.

As vigas foram colocadas sobre um apoio de primeira ordem e um de segunda ordem com dimensões de $10 \mathrm{~cm}$ de largura para ambos, dessa forma, em todas as vigas, o vão livre considerado foi $140 \mathrm{~cm}$. Os elementos foram todos ensaiados com cargas concentradas aplicadas nos terços médios.

Com o intuito de se obter os deslocamentos verticais na região central do vão, utilizaram-se dois equipamentos LVDT's (linear variation displacement transducer). Ambos ficaram posicionados um de cada lado da viga na parte central. As deformações específicas para o aço foram obtidas por meio de extensômetros elétricos aplicados na parte central da armadura metálica tracionada das vigas reforçadas VR1, VR2, VR3 e VRR3. Durante os ensaios, as deformações, forças e deslocamentos foram armazenados em um sistema de aquisição de dados e as aberturas de fissuras foram aferidas visualmente. A Figura 3 apresenta a configuração do ensaio realizado para as vigas.
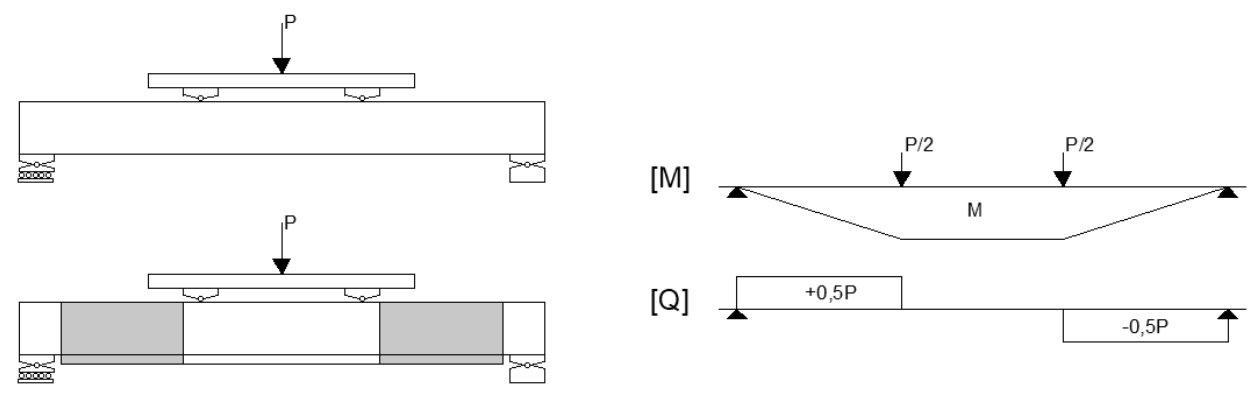

Figura 3 - Esquema do ensaio para as vigas

\section{ANÁLISE DOS RESULTADOS}

\subsection{Cargas últimas e modos de falha}

$\mathrm{Na}$ Tabela 3 são apresentados os valores das cargas últimas obtidas de forma analítica e pelo método experimental, assim como a capacidade de carga relativa às cargas teóricas e experimentais das vigas e como ocorreu o modo de falha. A exceção da viga VRR2, em que a camada de reforço descolou antes do concreto esmagar na zona comprimida, o modo de falha das vigas foi por esmagamento do concreto ocorrido após a armadura de aço, na zona tracionada, escoar. Todavia as armaduras de fibra de vidro não alcançaram a tensão de ruptura.

É válido salientar que, em todas as vigas, a capacidade de carga foi maior na verificação, que nos valores encontrados nos ensaios experimentais. A partir dos resultados apresentados anteriormente, pode-se observar que o aumento de carga foi mais significativo nas vigas reforçadas com barras de GFRP de 12 mm VR3 (97,92\%) e VRR3 (86,86\%) verificadas para ter um aumento de carga de $175,86 \%$. A diferença nos valores analíticos para os obtidos experimentalmente indica que somente a utilização dos princípios e das hipóteses do ELU não são a melhor alternativa de dimensionamento para essas estruturas. Por outro lado, com o aumento da seção das barras de reforço, a capacidade de carga das vigas aumentou. 


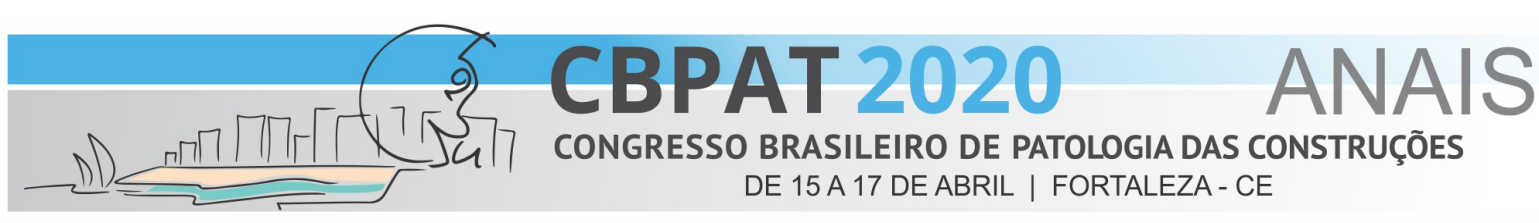

ISBN $978-65-86819-05-2$ ASSOCIACÃO BRASILEIRA

Tabela 3 - Capacidade de carga e modo de falha das vigas

\begin{tabular}{cccccc}
\hline Viga & \multicolumn{2}{c}{ Carga Última } & \multicolumn{2}{c}{ Capacidade de carga } & \\
& $\begin{array}{c}\text { Verificação } \\
(\mathbf{k N})\end{array}$ & $\begin{array}{c}\text { Experimental } \\
(\mathbf{k N})\end{array}$ & $\begin{array}{c}\text { Verificação } \\
\mathbf{\%}\end{array}$ & $\begin{array}{c}\text { Experimental } \\
\mathbf{\%}\end{array}$ & Modo de falha \\
\hline VT & 32,73 & 34,69 & - & - & Esmagamento do concreto \\
\hline VR1 & 66,59 & 55,49 & 103,45 & 59,96 & Esmagamento do concreto \\
\hline VR2 & 84,31 & 64,72 & 157,59 & 86,57 & Esmagamento do concreto \\
\hline VR3 & 90,29 & 68,66 & 175,86 & 97,92 & Esmagamento do concreto \\
\hline VT1 & 32,73 & 36,54 & - & - & Esmagamento do concreto \\
\hline VRR1 & 66,59 & 59,27 & 103,45 & 62,21 & Esmagamento do concreto \\
\hline VRR2 & 84,31 & - & 157,59 & - & Descolamento do reforço \\
\hline VRR3 & 90,29 & 68,28 & 175,86 & 86,86 & Esmagamento do concreto \\
\hline
\end{tabular}

A ancoragem das barras, com manta de fibra de vidro, aplicada na zona em que o esforço cortante é diferente de zero favoreceu para que não ocorressem falhas precoces na camada de reforço. É válido salientar que antes da aplicação da manta, as oito faces das vigas foram arredondadas para evitar a concentração de tensões nessas regiões, o que evitou o surgimento de ruptura nas laterais das vigas.

\subsection{Curva carga $x$ deslocamento no centro do vão}

As curvas carga $\mathrm{x}$ deslocamento mostram que o comportamento das vigas varia com o diâmetro da barra de fibra de vidro, sendo as curvas muito parecidas para cada configuração de reforço, a exceção das vigas reforçadas de $10 \mathrm{~mm}$, em que houve descolamento da camada de reforço na viga VRR2.

As vigas apresentaram comportamento semelhante antes da primeira fissura do concreto, entretanto com o aumento de carga, as vigas reforçadas se revelaram mais rígidas do que as vigas testemunho, como é possível observar na Figura 4.

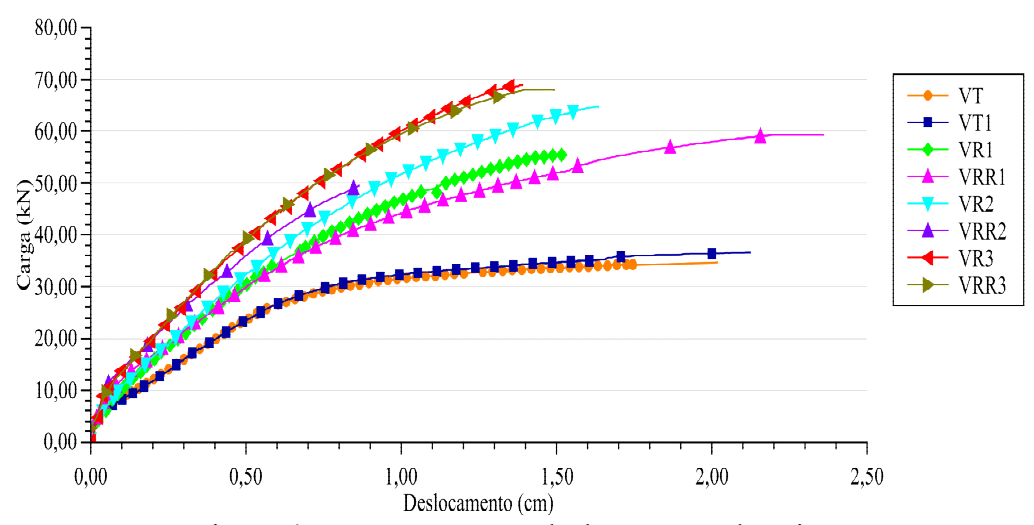

Figura 4 - Curvas carga $\mathrm{x}$ deslocamento das vigas

Na Tabela 4 são apresentados os valores das cargas de primeira fissura, as cargas de escoamento da armadura de aço, com seus respectivos deslocamentos, e a carga última das vigas. É possível observar que, para a primeira fissura, com o aumento do diâmetro das barras de fibra de vidro no reforço, o deslocamento vertical das vigas foi menor, entretanto essa relação não foi observada quando as armaduras de aço entraram em escoamento, assim como quando as vigas alcançaram a carga última. 
Tabela 4 - Valores das cargas de primeira fissura, escoamento do aço e cargas últimas das vigas

\begin{tabular}{|c|c|c|c|c|c|c|}
\hline \multirow[b]{2}{*}{ Vigas } & \multicolumn{2}{|c|}{ Primeira Fissura } & \multicolumn{2}{|c|}{ Escoamento do aço } & \multicolumn{2}{|c|}{ Carga última } \\
\hline & $\begin{array}{c}\text { Carga } \\
(\mathbf{k N}) \\
\end{array}$ & $\begin{array}{c}\text { Deslocamento } \\
(\mathrm{cm})\end{array}$ & $\begin{array}{c}\text { Carga } \\
(\mathrm{kN})\end{array}$ & $\begin{array}{c}\text { Deslocamento } \\
(\mathrm{cm})\end{array}$ & $\begin{array}{c}\text { Carga } \\
(\mathbf{k N}) \\
\end{array}$ & $\begin{array}{c}\text { Deslocamento } \\
(\mathrm{cm})\end{array}$ \\
\hline VT & 5,51 & 0,08 & 26,86 & 0,62 & 34,69 & 2,02 \\
\hline VR1 & 8,24 & 0,07 & 33,56 & 0,57 & 55,49 & 1,53 \\
\hline VR2 & 5,45 & 0,03 & 45,17 & 0,81 & 64,72 & 1,63 \\
\hline VR3 & 7,98 & 0,03 & 36,70 & 0,47 & 68,66 & 1,39 \\
\hline VT1 & 7,85 & 0,09 & 26,83 & 0,61 & 36,54 & 2,12 \\
\hline VRR1 & 8,01 & 0,05 & 41,71 & 0,88 & 59,27 & 2,36 \\
\hline VRR2 & 7,89 & 0,03 & 49,43 & 0,86 & - & - \\
\hline VRR3 & 8,01 & 0,04 & 36,86 & 0,46 & 68,28 & 1,49 \\
\hline
\end{tabular}

\subsection{Curva carga x deformação das vigas VR1, VR2, VR3 e VRR3}

As vigas VR1, VR2, VR3 e VRR3 receberam strain gages, que foram fixados na parte central da armadura metálica tracionada das vigas. Assim como mencionado anteriormente, durante os ensaios, as deformações foram armazenadas em um sistema de aquisição de dados. A Figura 5 apresenta os resultados obtidos no ensaio.

Verifica-se que para uma mesma carga, as vigas de maior diâmetro (VR3 e VRR3) apresentaram menores deformações que as demais. Para uma carga de $30 \mathrm{kN}$, a viga VR1 deformou 1,98\%o e as vigas VR2, VR3 e VRR3 1,85\%, 1,48\%o e $1,56 \%$ respectivamente.

A partir das curvas das vigas VR3 e VRR3 é possível verificar que suas deformações foram similares e suas rigidezes são maiores do que para as vigas VR1 e VR2.

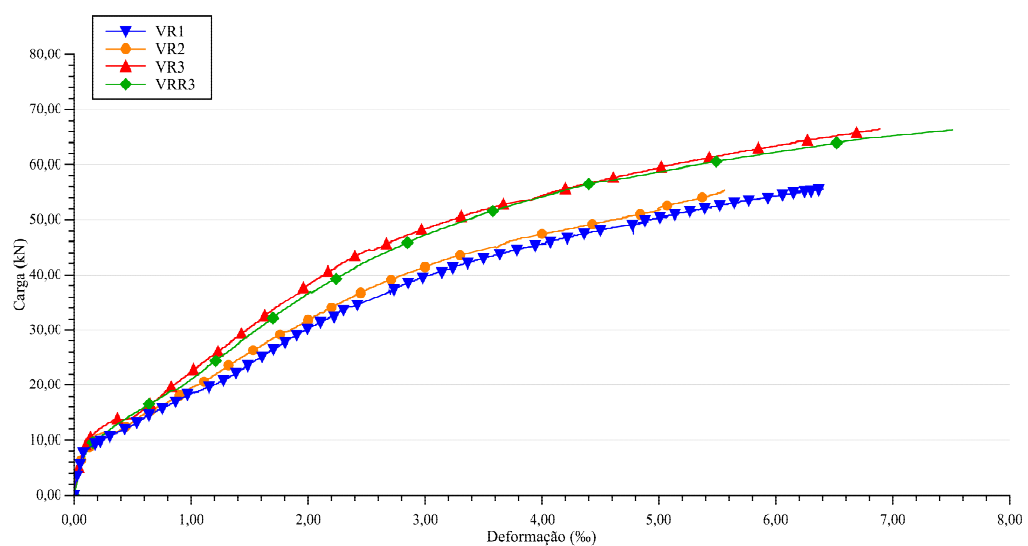

Figura 5 - Curvas carga x deformação das vigas VR1, VR2, VR3 e VRR3

\subsection{Análise de custo do reforço}

Por se tratar de um material relativamente novo no mercado brasileiro, seu custo elevado ainda é um aspecto importante que reflete no valor percebido no ramo da construção civil. O custo das barras de fibra de vidro é de R\$26,00/kg, podendo esse valor variar em função da demanda. Já as barras de aço são vendidas a R \$3,80/kg, ou seja, as barras de fibra de vidro custam aproximadamente sete vezes mais que às de aço. Deve-se, ainda, levar em consideração os custos da argamassa estrutural ( $\mathrm{R} \$ 4,60 / \mathrm{kg}$ ), da manta de fibra de vidro (R\$19,61/ $\mathrm{m}^{2}$ ) e das resinas utilizadas para fixá-la (aproximadamente primer R $\$ 280 / \mathrm{kg}$ e laminação R\$ 116/kg). A Tabela 5 apresenta o custo estimado de reforço por vigas de barras GFRP de mesmo diâmetro. 


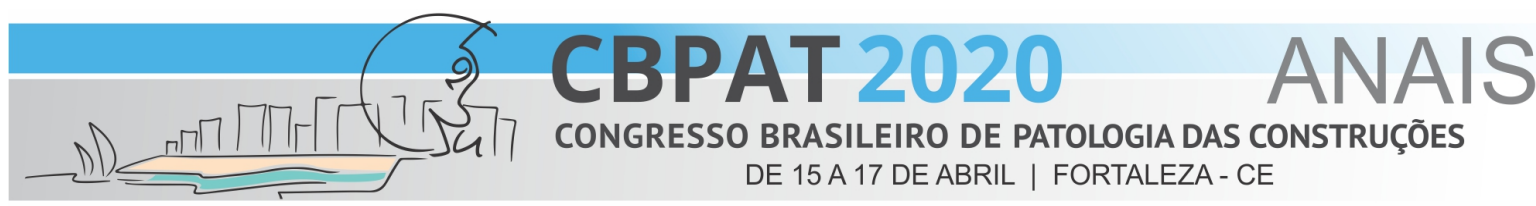
ISBN 978-65-86819-05-2 2 DES PATOLOCGIA BADAS CONSTRUÇOES

Tabela 5 -Custo do reforço e sua composição

\begin{tabular}{ccccccc}
\hline Vigas & $\boldsymbol{\Phi}_{\text {barras }}$ & Custobarras & Custoargamassa & Customanta & Custoresina & Custoreforço \\
\hline VR1/VRR1 & $8 \mathrm{~mm}$ & $\mathrm{R} \$ 6,50$ & $\mathrm{R} \$ 26,79$ & $\mathrm{R} \$ 7,55$ & $\mathrm{R} \$ 39,06$ & $\mathrm{R} \$ 79,90$ \\
\hline VR2/VRR2 & $10 \mathrm{~mm}$ & $\mathrm{R} \$ 13,00$ & $\mathrm{R} \$ 26,79$ & $\mathrm{R} \$ 7,55$ & $\mathrm{R} \$ 39,06$ & $\mathrm{R} \$ 86,40$ \\
\hline VR3/VRR3 & $12 \mathrm{~mm}$ & $\mathrm{R} \$ 13,00$ & $\mathrm{R} \$ 26,79$ & $\mathrm{R} \$ 7,55$ & $\mathrm{R} \$ 39,06$ & $\mathrm{R} \$ 86,40$ \\
\hline
\end{tabular}

A Tabela 6 apresenta o custo do reforço em relação à capacidade de carga, para cada diâmetro, das vigas que alcançaram cargas mais elevadas no ensaio. Como é possível verificar nas seções anteriores, essas vigas são a VRR1, VR2 e VR3.

Observa-se que o custo por capacidade tende a diminuir com o aumento do diâmetro das barras de GFRP. Para a viga VRR1, o custo por capacidade de carga foi aproximadamente $7,14 \%$ mais elevado que para a viga VR3 e foi praticamente o mesmo, quando comparado com a viga VR2. Essa relação ainda revela que, para as vigas, não compensou economicamente a adoção desse sistema de reforço, visto que o custo foi muito elevado, quando comparado à capacidade de carga alcançada.

Tabela 6 - Custo do reforço versus capacidade de carga das vigas VRR1, VR2 e VR3

\begin{tabular}{ccccc}
\hline Vigas & $\begin{array}{c}\text { Custoreforço } \\
(\mathbf{R S})\end{array}$ & $\begin{array}{c}\text { Capacidade de carga } \\
(\mathbf{k N})\end{array}$ & \%aumento & $\begin{array}{c}\text { Custoreforço/Capacidade } \\
\mathbf{( R S / k N )}\end{array}$ \\
\hline VRR1 & 79,90 & 59,27 & 62,21 & 1,35 \\
\hline VR2 & 86,40 & 64,72 & 86,57 & 1,33 \\
\hline VR3 & 86,40 & 68,66 & 97,92 & 1,26 \\
\hline
\end{tabular}

\section{CONCLUSÃO}

Os resultados obtidos experimentalmente revelam que o aumento do diâmetro das barras de GFRP proporcionou uma significativa elevação no incremento de carga, porém com valores mais baixos que os estimados analiticamente. Dessa forma, se reforça que a utilização dos princípios e das hipóteses do ELU não são a melhor alternativa de dimensionamento para essas estruturas, sendo de grande importância a utilização de concepções de dimensionamento específicas para esses materiais.

Em relação à ancoragem, a manta evitou que as barras escorregassem durante o ensaio e que a camada de reforço descolasse, à exceção da viga VRR2. De uma forma geral, esse sistema de ancoragem corroborou para o aumento da capacidade de carga das vigas.

A capacidade de carga das vigas apresentou uma elevação, principalmente para viga VR3 que chegou a aumentar $97,92 \%$ sua carga em relação à viga testemunho VT, entretanto foi aproximadamente $55,70 \%$ menor que o esperado na verificação analítica $(175,86 \%)$.

Todas as vigas reforçadas apresentaram um comportamento mais rígido que as vigas testemunho e essa rigidez se elevou com o aumento do diâmetro das barras de reforço.

Levando-se em conta os modos de falhas apresentados anteriormente, as barras de fibra de vidro não apresentaram um bom desempenho como reforço estrutural, devido ao concreto esmagar na zona comprimida após as armaduras positivas de aço escoarem e as barras de GFRP não chegarem à ruptura.

Além das condições ambientais, outro aspecto importante a ser considerado para a adoção desse sistema de reforço é o seu custo de produção, que ainda é inviável para execução em estruturas de grandes proporções. Porém, em estruturas expostas a altos níveis de agressividade ambiental, se torna uma boa opção de reforço.

O estudo realizado, de um modo geral, conseguiu obter resultados satisfatórios para a análise, contudo em função das cargas úlimas encontradas para as vigas reforçadas com barras de fibra de vidro apresentarem valores menores que os determinados de maneira teórica, ressalta-se a necessidade de um estudo mais específico para materiais GFRP.

\section{REFERÊNCIAS}

AMERICAN CONCRETE INSTITUTE. Guide for the design construction of externally bonded FRP systems for strengthening concrete structures: ACI 440.2R-02. Farmington Hills. 2002. 45p.

ASSOCIAÇÃO BRASILEIRA DE NORMAS TÉCNICAS. NBR 6118: Projeto de estruturas de concreto Procedimento. Rio de Janeiro. 2014. 
BEBER, A. J.; Comportamento estrutural de vigas de concreto armado reforçadas com compósitos de fibra de carbono. 2003. 317 f. Tese (Mestrado em Engenharia). Programa de Pós-Graduação em Engenharia Civil. Universidade Federal do Rio Grande do Sul. Porto Alegre. 2003.

COUTO, I. A.; Análise teórica e experimental do comportamento da aderência entre o concreto e barras de fibra de vidro impregnada por polímeros. 2007. 141f. Dissertação (Mestrado em Engenharia de Estruturas) - Escola de Engenharia de São Carlos da Universidade de São Paulo. São Paulo. 2007.

MENEGHETTI, L. C.; Análise do comportamento à fadiga de vigas de concreto armado reforçadas com PRF de vidro, carbono e aramida. 2007. 275f. Tese (Doutorado em Engenharia). Programa de Pós-Graduação em Engenharia Civil. Universidade Federal do Rio Grande do Sul. Porto Alegre. 2007.

NORA, J. B.; Análise experimental do Concreto Têxtil como reforço à flexão de vigas de concreto armado. 2018. 118f. Trabalho de Conclusão de Curso (Engenharia Civil). - Escola de Engenharia da Universidade Federal do Rio Grande do Sul. Porto Alegre. 2018.

PERUZZI, A. de P.; Comportamento das fibras de vidro convencionais em matriz de cimento Portland modificada com látex e adição de sílica ativa. 2002. 96f. Dissertação (Mestrado em Arquitetura) - Escola de Engenharia de São Carlos da Universidade de São Paulo. São Paulo. 2002.

PERUZZI, A. de P.; Estudo das alternativas do uso da fibra de vidro sem características álcali resistente em elementos construtivos de cimento Portland. 2007. 182f. Tese (Doutorado em Arquitetura) - Escola de Engenharia de São Carlos da Universidade de São Paulo. São Paulo. 2007.

TAVARES, H. D.; Análise teórica e experimental de vigas de concreto armadas com barras não metálicas de FRP. 2006. 118f. Dissertação (Mestrado em Engenharia Civil) - Escola de Engenharia de São Carlos da Universidade de São Paulo. São Paulo. 2006. 